\title{
Acreditación de aprendizajes por experiencia: el caso Escuela Ciencias de la Educación UNED Costa Rica
}

Adrián Solano Castro

Universidad Estatal a Distancia, Costa Rica; asolano@uned.ac.cr

\section{Resumen}

Este artículo trata sobre el proceso de acreditación de aprendizajes por experiencia en la Escuela Ciencias de la Educación de la Universidad Estatal a Distancia de Costa Rica. Se realizó una investigación de tipo documental con carácter descriptivo. El objetivo de la investigación fue analizar el proceso de acreditación del aprendizaje por experiencia para el período 1996-2012. Se obtuvo que la acreditación del aprendizaje por experiencia que ha favorecido el avance académico de los estudiantes y constituye un mecanismo de fortalecimiento de la educación permanente e inclusiva en la UNED.

Palabras clave: Educación Permanente No Formal, Educación Informal, Acreditación de aprendizajes, Educación Superior.

\begin{abstract}
Accreditation of experiential learning: the case of the faculty of education at UNED, Costa Rica

This article is about the process of accreditation of experiential learning in the School of Education Sciences at Distance Education University of Costa Rica. Documentary research was conducted in a descriptive way. The objective was to analyze the process of accreditation of experiential learning in the period 1996-2012. It was concluded that the accreditation of experiential learning has favored students in terms of academic progress, besides, it provides a mechanism for quality improvement and strengthening of permanent inclusive education at UNED.
\end{abstract}

Key words: Education, Accreditation of experiential learning, Permanent education, Non- formal and informal learning, Higher education.

flexibles y accesibles a todos los ciudadanos (Salinas, 2002; Casas, 2005; UNESCO, 2009).

La Universidad Estatal a Distancia (UNED) como parte de ese proceso busca formas de vinculación con la sociedad y sus diferentes sectores (productivo, laboral, empresarial, etc.). Una de las formas para fortalecer esta vinculación son los procesos de 
acreditación de aprendizajes por experiencia (AAE) cuya finalidad es proporcionar una oportunidad de ingreso, reingreso o avance en las carreras que ofrece la universidad (UNED, 1998).

Dentro de esta perspectiva la UNED enfrenta el reto que plantea Cieza 2006:

Es una necesidad evidente que las Instituciones de Educación Superior deben abrirse cada vez más a la sociedad y ofrecer posibilidades de interacción, intercambio y beneficio mutuo. Esta formación pretende ser una muestra de apertura a colectivos y personas (adultos, mayores y grupos con necesidades formativas especiales) que pueden verse beneficiadas de una acción formativa atrayente y enriquecedora a la vez, pero en cualquier caso favorecedora de un desarrollo personal y social (p. 2).

De igual forma Delors (1996) manifiesta que la educación superior puede contribuir a la educación permanente, al abrir sus puertas a los adultos que quieran reanudar los estudios, adaptar y enriquecer sus conocimientos, o satisfacer sus ansias de aprender en todos los ámbitos de la vida.

Frente a un panorama de retos y demandas para la universidad, las políticas sobre los procesos de AAE deben asumirse con responsabilidad en procura de resultados educativos de calidad. Además, con estrategias bien definidas que actúen como guías de estos procesos.

\section{- Referente teórico}

La reflexión, discusión y puesta en práctica de los procesos de AAE aunque no es nueva, recientemente ha estado vinculada a lineamientos de política educativa emitidos en el marco de la Unión Europea. Es así como se discute en diferentes Cumbres y Conferencias sobre la necesidad de articular dispositivos de acreditación de los aprendizajes no formales e informales, perfeccionando sus certificaciones más allá de las obtenidas en la formación académica.

Durante este siglo se suele definir como el origen del reconocimiento a los aprendizajes no formales a La Declaración de Copenhague (2002), mientras que la Conferencia de Bergen (2005) destaca la importancia de asegurar que el marco general de las cualificaciones del Espacio Europeo Educación Superior y el marco más amplio para el aprendizaje a lo largo de la vida sean complementarios, incluyendo la educación general y la formación profesional (González, s.f.).

Por una parte, en el caso de la UNED el marco de referencia son los procesos de educación permanente y de educación inclusiva. La educación permanente pensada como las experiencias de aprendizaje formal, no formal e informal en las diferentes etapas de la vida (Rama, 2006; Silveira, 1998). Por lo tanto, la AAE en la UNED tiene su fundamento en los procesos de educación permanente de la manera como la conceptualiza Tünnermann Bernheim (1995):

La educación permanente demanda de las instituciones de educación superior el reconocimiento de que es posible adquirir conocimientos y destrezas superiores en instituciones que no necesariamente han sido reconocidas como entidades educativas, como por ejemplo las empresas (p. 14).

Por otra parte, está presente la idea de educación inclusiva vista como una forma amplia de educación para todos, que transciende ámbito de la institución educativa y de la educación porque supone un pensamiento social transformador y la igualdad de oportunidades en sociedades más democráticas (Ainscow \& Miles, 2008; De la Puente, 2009; Parrilla, 2002).

El concepto de AAE debe comprenderse en un sentido abarcador, es decir, se trata de un proceso de evaluación curricular en el que mediante un peritaje de atestados, se reconocen determinadas competencias y a continuación, se certifican esas competencias con base en un determinado plan de estudios. Por lo tanto, la UNED visualiza los procesos de AAE como mecanismos de flexibilidad curricular que permite fomentar oportunidades de movilidad estudiantil y ofrecer caminos de formación a los estudiantes de acuerdo con sus intereses, expectativas y necesidades académicas (Gómez \& Celis, 2005).

Relacionado con la temática de flexibilidad curricular puede encontrarse en una amplia variedad 
de trabajos de investigación educativa en el área curricular (Didriksson, 2010; Nieto \& Díaz, 2005; Escalona, 2008; Pedroza, 2000), lo que ha dado origen a diversas interpretaciones sobre el tema; para el propósito de la investigación abordamos la flexibilidad curricular como la presenta Elligton (1997) citado por Villa (2002) cuando plantea por formación flexible:

(...) un término genérico que cubre todas aquellas situaciones donde los aprendices tienen algo que decir en relación con cómo, dónde y cuándo sus aprendizajes pueden tener lugar, ya sea dentro del contexto de la institución tradicional centrada en las materias o en contextos no tradicionales tales como el aprendizaje abierto, la educación a distancia, los cursos de amplio acceso, o la educación profesional continuada (p. 33).

En síntesis, la AAE en la UNED es un proceso académico de evaluación de las experiencias con valor educativo de un estudiante, en el cual se aprecian factores de educación no formal o informal, así como su experiencia en el mercado laboral. De manera que el énfasis está en su experiencia laboral y no en el deber ser del plan de estudios.

\section{METODOLOGÍA}

El método de investigación fue de carácter documental porque se analiza y presentan documentos del Programa de Acreditación por Experiencia. También es de tipo exploratorio, porque es una aproximación a una realidad poco trabajada y explorada en la universidad. Además, contiene elementos de tipo descriptivo, pues se trata de describir características del fenómeno de la $\mathrm{AAE}$ en las carreras de Ciencias de la Educación.

La información que se presenta en esta investigación documental fue ofrecida por el programa de AAE y la Oficina de Registro de la UNED. Se consultaron 69 expedientes de acreditación presentados por estudiantes de las carreras de la Escuela de Educación así como el Sistema de Administración de Estudiantes (SAE), además, de los documentos de reconocimiento de créditos de la UNED.
Para desarrollar el estudio, la población estuvo comprendida por los estudiantes que solicitaron AAE en las carreras de la Escuela de Educación para el período 1996-2012. En una primera fase, se consultaron los expedientes de los estudiantes que solicitaron AAE, así como el SAE. En una segunda fase, se efectuó un trabajo predominantemente descriptivo, del proceso de acreditación por experiencia con la finalidad de analizar el comportamiento en las carreras de la Escuela de Ciencias de la Educación.

El análisis de datos secundarios se llevó a cabo con estadísticas descriptivas y consistió en la descripción de los datos; por medio de gráficos y cuadros en las que se puede apreciar claramente el comportamiento y las tendencias del fenómeno de la AAE en la Escuela Ciencias de la Educación.

\section{RESULTADOS}

Se presentan a continuación los datos cuantitativos derivados del proceso de acreditación del aprendizaje por experiencia en la Escuela Ciencias de la Educación de la UNED, clasificados por especialidad para el período comprendido entre 1996-2012.

De la totalidad de solicitudes de acreditación por experiencia se observa que la mayor cantidad corresponden a mujeres $74 \%$ mientras que los hombres el $26 \%$ como lo muestra la figura 1 .

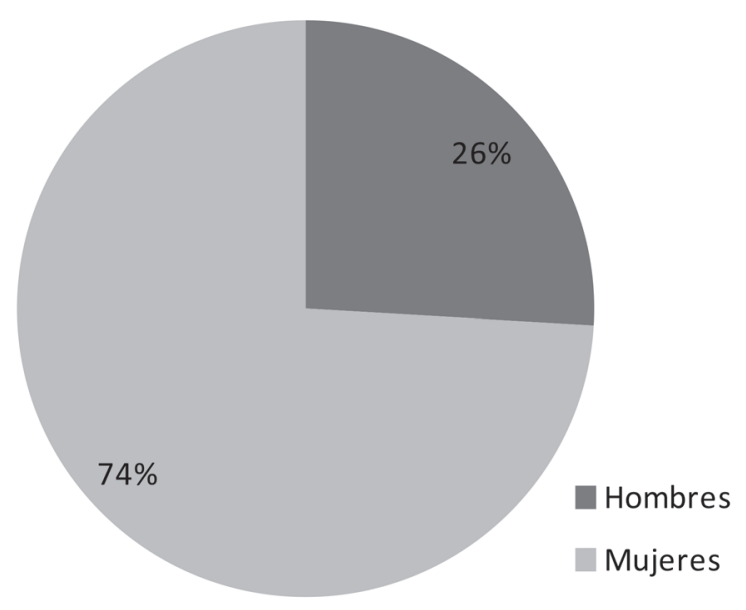

Figura 1. Estudiantes que solicitaron acreditación por experiencia Escuela Ciencias de la Educación UNED (1996-2012). Fuente: Elaboración Propia. 
TABLA 1

Solicitudes de acreditación por experiencia Escuela Ciencias de la Educación UNED (1996-2012)

\begin{tabular}{lcc}
\multicolumn{1}{c}{ Carrera } & Número de solicitudes \\
\hline Educación Preescolar & N & $\%$ \\
Administración Educativa & 2 & 2.84 \\
I y II Ciclos & 4 & 5.71 \\
Educación Especial & 4 & 5.71 \\
Informática Educativa & 10 & 14.30 \\
Total & 49 & 70.00 \\
\hline
\end{tabular}

Fuente: Elaboración Propia.

La tabla 1 muestra la distribución de solicitudes de acreditación de los estudiantes de las carreras de la Escuela de Educación durante el período estudiado. Hay una gran concentración de solicitudes en la carrera Informática Educativa el $70 \%$, mientras que la carrera de Educación Especial tiene un $15 \%$ entre las dos abarcan el $85 \%$ de las solicitudes de AAE.

En la tabla 2 se puede evidenciar que de la totalidad de solicitudes recibidas 69 , la mayor cantidad de
AAE recaen en asignaturas del área de informática siendo Lenguaje Logo $1 \quad 29$ \% la que más solicitudes recibió, después Introducción a la Computación $18.85 \%$ y Aplicaciones de la Informática a la Educación $17.40 \%$, que en conjunto tienen el 65\% de las AAE tramitadas durante este período. Asimismo se destaca que las demás asignaturas se comportan de manera regular en el rango de cuatro a una acreditación solicitada.

Las solicitudes de AAE de acuerdo con los datos parecen en su mayoría ser dictaminadas como positivas, en la figura 2 se puede evidenciar que la totalidad de solicitudes de AAE 69, un porcentaje muy alto es aprobado $94 \%$ mientras que solamente un 6 $\%$ de las solicitudes son denegadas.

\section{DISCUSIÓN DE RESULTADOS}

Con el propósito de conocer el fenómeno de la AEE en la Escuela Ciencias de la Educación se discuten y analizan los resultados obtenidos, así como las conclusiones del estudio. Además, se mencionan

TABLA 2

Solicitudes de acreditación por experiencia en asignaturas de Escuela Ciencias de la Educación UNED (1996-2012)

\begin{tabular}{lcc}
\multicolumn{1}{c}{ Asignatura } & Solicitudes de Acreditación & \% \\
Lenguaje Logo 1 & 20 & 29.00 \\
Introducción a la Computación & 13 & 18.85 \\
Aplicaciones de la Informática en Educación 1 & 12 & 17.40 \\
\hline Investigación Educativa & 4 & 5.80 \\
Relaciones Familiares Profesionales y Comunales & 3 & 4.34 \\
Alumnos con Necesidades Educativas Especiales & 3 & 4.34 \\
Material Didáctico para Educación Especial & 2 & 2.89 \\
Técnicas y Estrategias p/ Manejo de Grupos Centro Escolar & 2 & 2.89 \\
Aplicaciones de la Informática en Educación 2 & 2 & 2.89 \\
Introducción a la Educación Especial & 1 & 1.45 \\
Inglés para Educadores de Preescolar & 1 & 1.45 \\
Música para I y II Ciclos & 1 & 1.45 \\
Práctica Docente & 1 & 1.45 \\
Comunicación Escrita & 1 & 1.45 \\
Recursos Naturales & 1.45 \\
Primeros Auxilios & 1.45 \\
Comportamiento Organizacional & 1.45 \\
Total & 1 & 1 \\
\hline
\end{tabular}

Fuente: Elaboración Propia. 


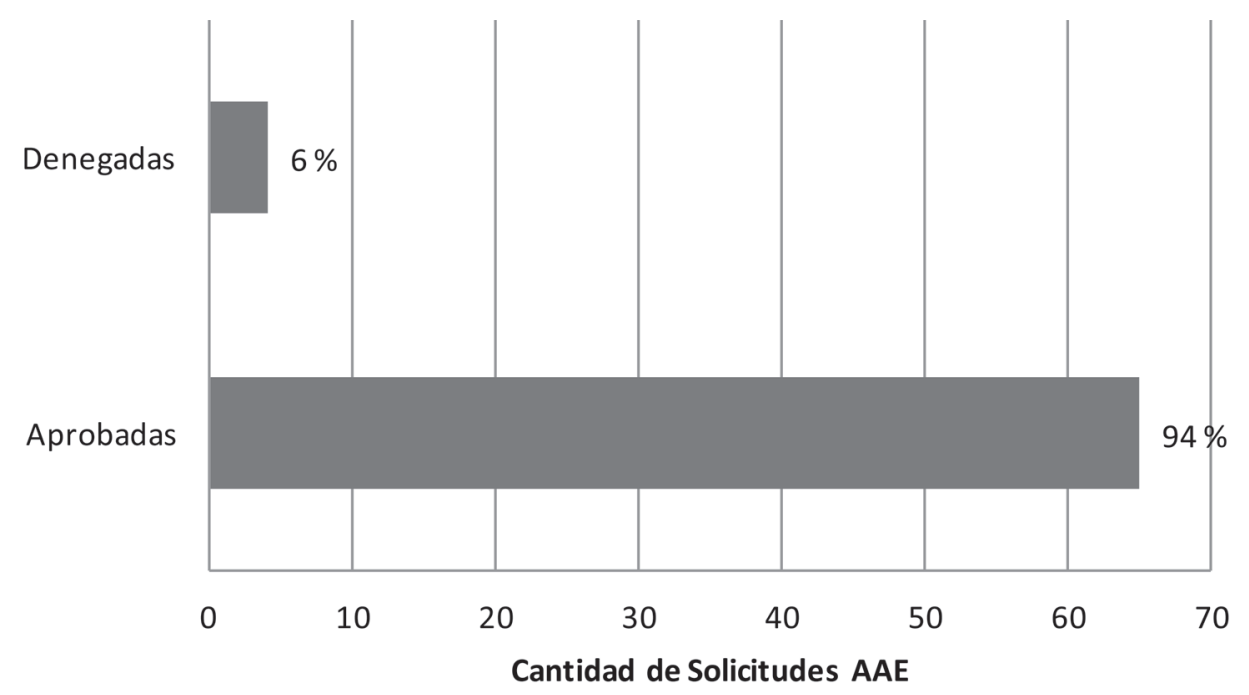

Figura 2. Tipo de resultado de las solicitudes de acreditación por experiencia Escuela Ciencias de la Educación UNED (1996-2012). Fuente: Elaboración Propia.

aquellas limitaciones evidenciadas en el desarrollo del proceso investigativo y se proponen recomendaciones para la elaboración de investigaciones futuras.

Los resultados de la investigación mostraron que las solicitudes de acreditación en las carreras de la Escuela de Educación las realiza una población mayoritariamente femenina, aproximadamente un $75 \%$ mujeres y $25 \%$ hombres; esta condición concuerda con algunos estudios (Brenes 2005; Vargas 2012), que concluyen que en nuestro país la mayoría de mujeres continua estudiando carreras tradicionalmente tipificadas como femeninas entre ellas las de educación.

También, los resultados obtenidos indican que en una gran mayoría de las carreras de la Escuela de Educación hubo solicitudes de AAE, situación que concuerda con los planteamientos de Medina y Sanz (2011); Paya (2010) que identifican los procesos de AAE como una práctica educativa que viene en aumento. Además, permite ver de manera concreta la aspiración de la UNED por contribuir en la construcción de una sociedad costarricense más democrática basada en el acceso al conocimiento.

De igual modo, sobre el papel de la AAE como mecanismo de flexibilidad curricular, se encuentra congruencia entre los resultados del estudio y el planteamiento de Villa (2002) cuando manifiesta que:

Desde el punto de vista institucional, la formación flexible se refiere a la gama de formas o medios, apoyos, tiempos y espacios que una institución ofrece para responder a las demandas de formación y para generar, igualmente, una mayor cobertura y calidad del servicio educativo (p. 32).

Los aspectos propuestos por Villa (2002) se han comprobado en la investigación, porque la UNED ofrece un programa orientado hacia el reconocimiento de aprendizajes adquiridos en contextos no formales e informales. Además, del análisis descriptivo se desprende que un alto porcentaje de las solicitudes fueron aprobadas $96 \%$. Este porcentaje de aprobación permite afirmar que la AAE se constituye en un mecanismo flexibilidad curricular, es decir, los estudiantes disponen de otras opciones para reconocer asignaturas del plan de estudios.

Entre los hallazgos más significativos que se derivan del análisis de la información obtenida, es que los estudiantes de la carrera de Informática Educativa presentaron el $70 \%$ de las solicitudes de AAE, esta condición podría estar asociada con el trabajo del personal de la Fundación Omar Dengo con el Programa de Informática Educativa 
(PIE-MEP-FOD) que surgió en la década de los 80 para orientar en forma directa a los profesores del Programa de Informática Educativa para el primer y segundo ciclo de la educación formal pública costarricense (Fonseca, 2000; Portilla, 2001).

En congruencia con el planteamiento anterior los estudiantes de la carrera Informática Educativa presentaron más solicitudes de AAE en la asignatura Lenguaje Logo 1; esta situación se podría explicar por la labor del personal de la Fundación Omar Dengo que impulsó un proyecto que se concibió como de carácter nacional y fue la capacitación de maestros sobre el uso de la computadora y el programa Logo en el aula (Víquez, 2008). Por consiguiente, muchos de los estudiantes que ingresaron a la carrera Informática Educativa contaban con la capacitación y experiencia profesional situación que parecería les permitió acreditar por experiencia dicha asignatura.

Por una parte, es importante destacar que los resultados del estudio demuestran que las asignaturas de informática con un componente altamente práctico representan más del $60 \%$ de las solicitudes de AAE, además con un alto porcentaje de aprobación. Esta situación pareciera estar asociada a la idea de aprendizaje situado, entendido como el aprender haciendo donde la actividad real permite que los estudiantes desarrollen las habilidades y destrezas que definitivamente son equiparables con la enseñanza universitaria.

Por otra parte, aunque porcentualmente en menor medida se pueden identificar algunas asignaturas de la carrera Educación Especial con una condición similar, a las que presentan las de la carrera Informática Educativa anteriormente comentadas. Esta situación podría obedecer a los procesos de capacitación para profesores de primaria en materia de educación especial durante las décadas de los 60 y 70 (Meléndez, 2007). Estos procesos permitieron actualizar al maestro de primaria en alguna necesidad educativa especial, por consiguiente, en el mercado laboral se encuentran maestros de primaria con experiencia específica en educación especial; situación que parece indicar les permitió acreditar por experiencia asignaturas del plan de estudios de la carrera de dicha especialidad.
Entre los hallazgos del estudio realizado respecto de las solicitudes de AAE presentadas por los estudiantes de las otras carreras de la Escuela de Educación, a saber: (a) Administración Educativa; (b) Preescolar; y (c) I y II Ciclos. Sobresalió que los estudiantes solicitaron AAE de asignaturas que pertenecen a la formación complementaria en el plan de estudios tales como Introducción a la Computación e Inglés para Preescolar. Esta situación podría interpretarse como que la oferta educativa en el país tanto formal como no formal, ofrece cursos en los campos de la computación y el inglés equiparables con la formación universitaria.

En la misma línea, como lo plantea Solano, (2014) sobre la formación en computación

(...) el mercado exige conocimientos en computación para los diferentes perfiles ocupacionales, por consiguiente, la oferta educativa tanto formal como no formal proporciona cursos básicos de computación los cuales se han ofertado con el objetivo de lograr una inserción exitosa en el mercado laboral. De manera que la asignatura Introducción a la Computación contemplada en la malla curricular, ha posibilitado que los estudiantes soliciten la AAE, porque las competencias en elementos de computación las desarrollaron en algún tramo de su formación o la adquirieron en el campo laboral (p. 77).

Además, este resultado es consistente con planteamientos teóricos sobre los sistemas de AAE propuestos por autores (Medina \& Sanz, 2009; Delors, 1996) que han indicado la necesidad de un replanteamiento de los métodos tradicionales de titulación donde se contemplen los conocimientos adquiridos por caminos alternativos de educación.

En lo que corresponde a las solicitudes de AAE presentadas por los estudiantes que fueron dictaminadas como denegadas; el porcentaje de $6 \%$ no aprobado resulta consistente con lo propuesto el autor (Medina \& Sanz, 2011) sobre la posibilidad real de éxito de los procesos de AAE. También, por el tipo de evidencias que suministraron los estudiantes para los expedientes de AAE, pareciera que desconocían los procedimientos académicos y administrativos que rigen la AAE en la UNED. 


\section{CONCLUSIONES}

Es posible afirmar, que el fenómeno de la $\mathrm{AAE}$ en la Escuela Ciencias de la Educación de la UNED durante el período estudiado, tuvo un comportamiento regular, marcado por una concentración de solicitudes en la carrera Informática Educativa. Específicamente en las asignaturas del plan de estudios con un alto componente práctico.

Los resultados revelan que los estudiantes lograron obtener una mayor cantidad de acreditaciones por experiencia en la asignatura Lenguaje Logo 1, por lo que se puede concluir que para el período estudiado en el campo de la Informática Educativa los cursos de formación continua fueron equiparables con la formación universitaria.

Otra conclusión general es que la AAE es una opción real para los estudiantes que permite el avance en el plan de estudios. Es relevante observar que de la totalidad de casos analizados, un 96 \% recibió un dictamen positivo en su solicitud.

Es importante destacar que este comportamiento permite conceptualizar a la AAE como un mecanismo de flexibilidad curricular, porque logra romper con la concepción rígida y tradicional del currículo disciplinar que prevalece en la institución.

De acuerdo con los expedientes analizados y particularmente por los dictaminados negativos, se podría interpretar que hay desconocimiento del proceso de AAE por parte de estudiantes, a pesar de que el Programa de Acreditación cuenta y difunde información que explicita que es la $\mathrm{AAE}$, así como los procedimientos por seguir para solicitar un estudio de AAE.

Es importante destacar que la evaluación de las solicitudes de AAE es un fenómeno complejo, los datos aquí mostrados deben ser tomados como resultados iniciales y no como indicadores concluyentes, debido a las características del método de investigación empleado, la AAE es un proceso de múltiples factores que no fueron abordados en el presente estudio.

Finalmente con respecto al proceso de investigación presenta limitaciones que apuntan algunas vías para investigaciones futuras, por ejemplo, se propone analizar los procedimientos de evaluación de los expedientes de AAE, para tratar de descubrir mecanismos que fortalezcan el proceso de evaluación de estos últimos, además es importante el análisis de la AAE en el contexto de los diseños curriculares en la institución y finalmente resultaría importante conocer la opinión de los estudiantes con respecto a los procesos de AAE, estas posibilidades permitirían profundizar en el fenómeno de la AAE.

\section{REFERENCIAS}

Ainscow, M., \& Miles, S. (2008). Por una educación para todos que sea inclusiva: ¿ Hacia dónde vamos ahora? Perspectivas, 38(1), 17-44.

Brenes, I. (2005). Los géneros en la educación superior universitaria en Costa Rica. Rosaura Sierra y Gisela Rodríguez (Comp.), Feminización de la Matrícula de Educación Superior en América Latina y El Caribe, México, UDUAL/UNESCO Instituto para la Educación Superior en América Latina y El Caribe, IESALC.

Casas Armengol, M. (2005). "Nueva universidad ante la sociedad del conocimiento» [artículo en línea]. Revista de Universidad y Sociedad del Conocimiento (RUSC) (Vol. 2, No 2). UOC. Recuperado el 29 de julio de 2014, de http://www.uoc.edu/rusc/2/2/dt/esp/ casas.pdf

Cieza, J. A. (2006). E-learning factors. A lifelong learning challenge inside the European Space for Higher Education framework. In Paper presented at the Virtual Campus 2006 Post-proceedings. Selected and Extended Papers-VC'2006. CEUR Workshop Proceedings, Available at: http://CEUR-WS. org (Vol. 186).

De la Puente, J. L. (2009). Hacia una Educación Inclusiva para todos. Revista Complutense de Educación, 20(1), 13-31.

Delors, J. (1996). La educación o la utopía necesaria. Informe a la UNESCO de la Comisión Internacional sobre la Educación. La educación encierra un tesoro, 13-36.

Didriksson, A. (2010). La construcción curricular: innovación, flexibilidad y competencias. Educación Superior y Sociedad, 10 (2), 29-52. 
Escalona, L. (2008). Flexibilidad curricular: elemento clave para mejorar la educación bibliotecológica. Investigación bibliotecológica, 22 (44), 143-160.

Fonseca, C. (2000). Maestros Innovadores: El Papel de la Informática Educativa en el Desarrollo Profesional de los Educadores Costarricenses. Informática Educativa, 13 (2), 163-185.

Gómez, V.M. \& Celís, J.E. (2005). Factores de innovación curricular y académica en la educación superior. Uni-pluri/versidad Vol $5 \mathrm{~N} 1$ facultad educación universidad de Antioquia Medellín Colombia.

González, T. D. "El reconocimiento de la competencia profesional desde las organizaciones". Sentido de la cooperación de universidad y empresa. Desafíos e solucións dende a Orientación Profesional para a certificación da competencia profesional, 31 .

Medina, O. \& Sanz, F. (2009). Los sistemas de reconocimiento y acreditación de los aprendizajes no formales e informales: referencias históricas, funciones socioeducativas y perspectiva teórica. Revista de Educación, 348, 253-281.

Meléndez, L. (2007). La Educación Especial en Costa Rica. Fundamentos y Evolución. San Jose, Costa Rica EUNED.

Nieto, L.D. \& Díaz, M. (2005). La formación de profesores universitarios para la flexibilidad curricular mediante estrategias virtuales. En: Duart, Josep M.; Lupiáñez, Francisco (coords.). Las TIC en la universidad: estrategia y transformación institucional. [monográfico en línea]. Revista de Universidad y Sociedad del Conocimiento (RUSC). Vol. 2, núm. 1. UOC. Recuperado 29 julio de 2014 de http:// www.uoc.edu/rusc/dt/esp/nieto0405.pdf> ISSN 1698-580X

UNESCO. (2009, junio). Conferencia Mundial sobre la Educación Superior 2009. La nueva dinámica de la Educación superior y la investigación para el cambio social y el desarrollo. Recuperado el 29 de julio del sitio web de la UNESCO: http://www.unesco.org/ education/WCHE2009/comunicado_es.pdf

Parrilla, Á. (2002). Acerca del origen y sentido de la educación inclusiva. Revista de educación, (327), 11-29.

Paya, A. (2010) Políticas de educación inclusiva en América Latina. Propuestas, realidades y retos de futuro. Revista Educación Inclusiva 3 (2), 125-142.
Pedroza, R. (2000). El currículo flexible en el modelo de universidad organizado en escuelas y facultades. Revista de la educación superior, Núm. 117, eneromarzo, anuies, México, 2000.

Portilla, M. (2001). Tecnología informática y Educación de Adultos: El caso de los cursos de navegación por internet y correo electrónico para adultos mayores y ciudadanos de oro de la Fundación Omar Dengo. Espacios Revista Centroamericana de Cultura Política N13.

Rama, C. (2006). La tercera reforma de la educación superior en América Latina. Buenos Aires: Fondo de cultura económica.

Salinas, J. (2002). Modelos Flexibles como respuesta de las universidades a la sociedad de la información. Acción Pedagógica, 11 ( 1), 4-13.

Silveira, S. (1998). La educación para el trabajo: un nuevo paradigma. Seminario Taller La educación como instrumento para superar la pobreza y el desempleo. Secretaría Pro Tempore del Grupo de Río. Ciudad de Panamá, 5-7.

Solano, A. (2014). Acreditación de Aprendizajes por Experiencia como Factor de la Calidad en UNED. La Experiencia de la Escuela Ciencias de la Administración. Revista Calidad en Educación Superior, 5 (1), 64-83.

Tünnermann Bernheim, C. (1995). La Educación Permanente y su Impacto en la Educación Superior, en Nuevos documentos sobre educación superior. (París: Unesco).

Universidad Estatal a Distancia. (2008). Reglamento de Acreditación Aprendizajes por Experiencia. San José, C.R.: Consejo Universitario.

Vargas, I. (2012). La perspectiva de género desde el estudiantado de práctica supervisada de la carrera de Orientación en la Universidad Nacional (UNA). Revista Electrónica Actualidades Investigativas en Educación, 12(2), 1-16.

Villa, M. D. (2002). Flexibilidad y Educación Superior en Colombia. Icfes.

Víquez, M. (2008). Las Tecnologías de información y comunicación (TICs) como respuesta a necesidades educativas del medio rural. Revista Electrónica Educare, 12, 121-142. 